\title{
Successful Simultaneous Endovascular Repair of Traumatic Portal Vein Pseudoaneurysm and Aortic Injury
}

\author{
Seon Uoo Choi, M.D.*, Seon Hee Kim, M.D.*, Sung Jin Park, M.D.*, Chan Ik Park, M.D.*,
} Up Huh, M.D. ${ }^{\dagger}$, Seunghwan Song, M.D. ${ }^{\dagger}$, Chang Won Kim, M.D. ${ }^{\ddagger}$, Hyuk Jin Choi, M.D. ${ }^{\S}$

${ }^{*}$ Department of Trauma Surgery, Pusan National University Trauma Center, ${ }^{\dagger}$ Department of Thoracic and Cardiovascular Surgery, Pusan National University School of Medicine, Biomedical Research Institute, Pusan

National University Hospital, ${ }^{\ddagger}$ Department of Radiology, Pusan National University School of Medicine,

Biomedical Research Institute, Pusan National University Hospital, ${ }^{\S}$ Department of Neurosurgery and Medical Research Institute, Pusan National University Hospital, Pusan National University School of Medicine, Busan, Korea
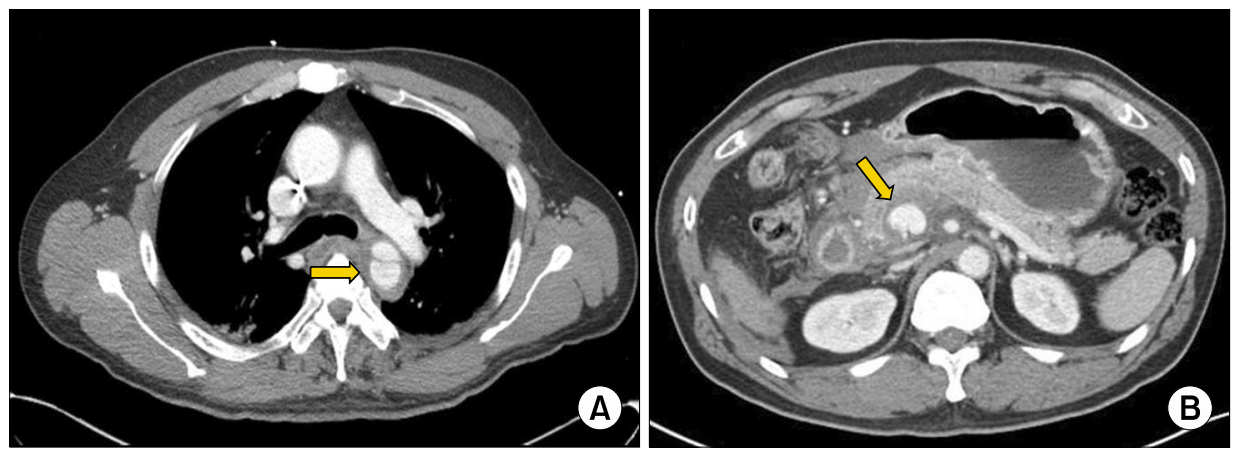

Fig. 1. Initial computed tomography (CT) scan. (A) Contrast enhanced CT scan of the chest revealed a 3 $\mathrm{cm}$ long pseudoaneurysm at the isthmus of the aorta (arrow) and intramural hematoma in the descending thoracic aorta. (B) Venous phase image of the abdominal CT scan demonstrates a $2 \mathrm{~cm}$ portal vein aneurysm (arrow) and hematoma around the pancreas head.

A 50-year-old man visited to the trauma center after driver traffic accident. Multiple injuries were found on computed tomography (CT) scan including aortic injury, duodenal perforation, pancreas contusion, and portal vein aneurysm (Fig. 1). Exploratory laparotomy was performed immediately for control of bleeding, primary repair of perforated duodenum, transverse colon and stomach. As soon as the operation was completed, the patient was transferred to the angio-room. The endovascular repair for aorta and portal vein was performed (Fig. 2). The patient was

Received September 18, 2018,

Revised October 1, 2018

Accepted October 1, 2018

Correspondence to: Seon Hee Kim, M.D.

Department of Trauma Surgery, Pusan

National University Hospital, 179

Gudeok-ro, Seo-gu, Busan 49241, Korea

Tel: +82-51-240-7369, Fax: +82-51-240-7719,

E-mail: ksh810427@naver.com

ORCID:

http://orcid.org/0000-0002-9756-9652 discharged from the hospital 3 months later, without disability and complication. At 1-year follow up CT scan revealed no endoleak and good patency of stent-graft (Fig. 3).

Traumatic aortic injury (TAI) is the second most common cause of death from blunt trauma. Paradigm of TAI treatment has changed from open surgery to endovascular repair because of the benefits of minimal invasiveness and avoidance of full heparinization in multiple trauma patients [1]. Although pseudoaneurysm of the post-traumatic portal

Copyright (C) 2018 by Korean Society of Acute Care Surgery

(c) This is an Open Access article distributed under the terms of the Creative Commons Attribution Non-Commercial License (http://creativecommons.org/licenses/by-nc/4.0) which permits unrestricted non-commercial use, distribution, and reproduction in any medium, provided the original work is properly cited. 

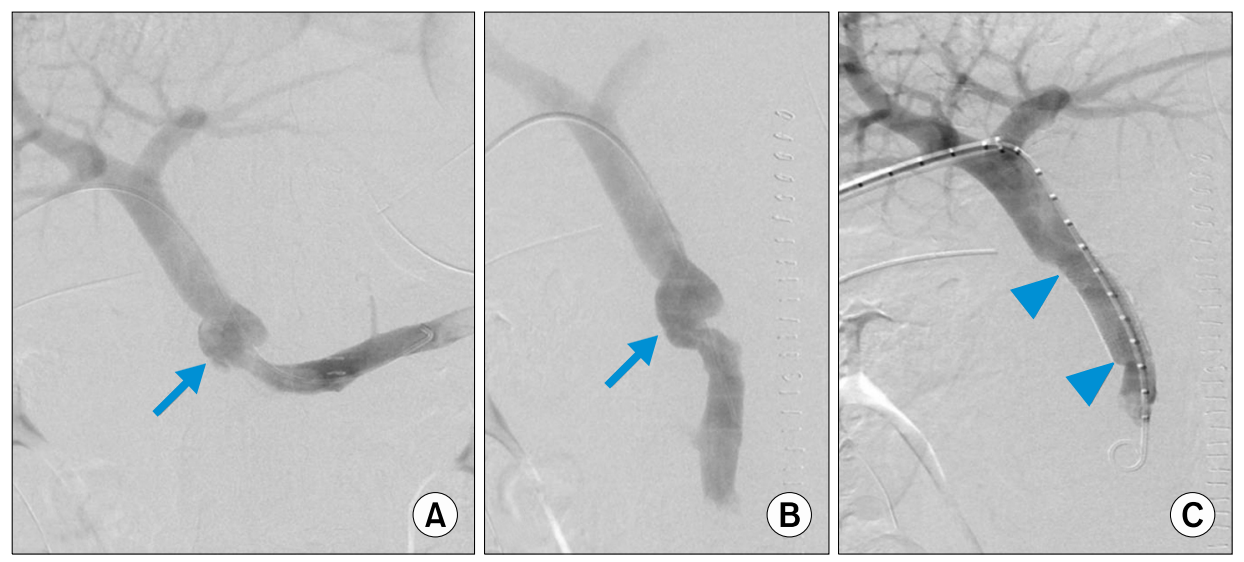

(C)
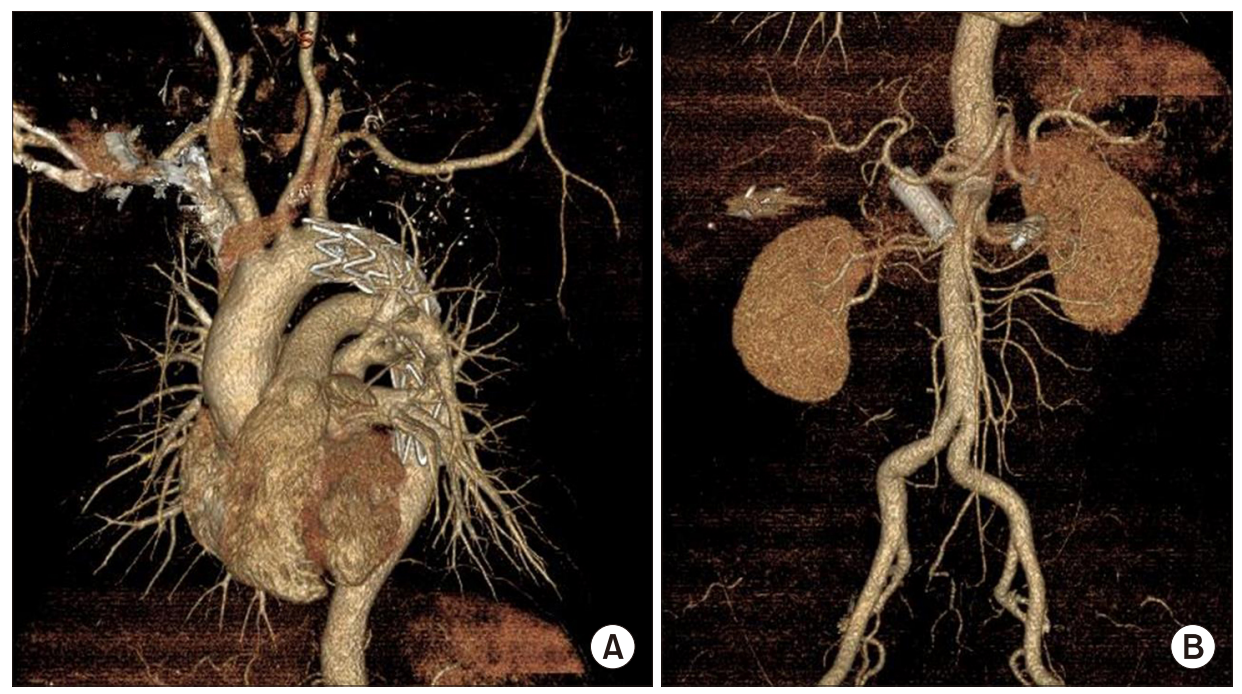

Fig. 3. Three-dimensional aorta angiography 1-year postoperative. (A) Transendovascular aortic repair indicated a complete resorption of the traumatic aortic dissection. (B) Stent graft insertion in the portal vein indicated good patency and no endoleak. Embolization of the traumatic pseudoaneurysm of the midcolic artery showed no extravasation or bowel ischemic change.

vein rarely occurs, surgical mortality is also high because of the difficulty in maintaining hemostasis and the secondary damages associated with enteric and pancreatic injuries [2,3]. Combined angiographic and surgical approaches could provide advantages for exsanguinating patients with multiple injuries and especially for management of major vascular, high-grade liver, or pelvic bleeding $[4,5]$. We achieved a good result through combined endovascular open surgical treatment in the patient who required the management of damage control resuscitation.

\section{Acknowledgments}

This work was supported by clinical research grant from Pusan National University Hospital in 2017.

\section{Conflicts of Interest}

No potential conflict of interest relevant to this article was reported. 


\section{References}

1. Ball CG, Kirkpatrick AW, D'Amours SK. The RAPTOR: resuscitation with angiography, percutaneous techniques and operative repair. Transforming the discipline of trauma surgery. Can J Surg 2011;54:E3-4.

2. Pachter HL, Drager S, Godfrey N, Lefleur R. Traumatic injuries of the portal vein. The role of acute ligation. Ann Surg 1979;189: 383-5.

3. Laopaiboon V, Aphinives C, Pugkem A, Thummaroj J, Puttharak W, Soommart Y. Selective transcatheter embolization for treatment of post-traumatic hepatic artery and portal vein pseudoaneurysms. J Med Assoc Thai 2006;89:248-52.

4. Hoffer EK, Borsa JJ, Bloch RD, Fontaine AB. Endovascular techniques in the damage control setting. Radiographics. 1999;19: $1340-8$.

5. Gruen RL, Brohi K, Schreiber M, Balogh ZJ, Pitt V, Narayan M, et al. Haemorrhage control in severely injured patients. Lancet 2012;380:1099-108. 